\title{
Correlative Light and Electron Microscopy (CLEM) for Characterization of Lithium Ion Battery Materials
}

\author{
Ch. Thomas, ${ }^{*}$ M. Edelmann, ${ }^{*}$ D. Lysenkov, ${ }^{* *}$ C. Hafner, ${ }^{* * *}$ T. Bernthaler, ${ }^{* * *}$ and G. Schneider*** \\ * Carl Zeiss AG, Corporate Research and Technology, 73446 Oberkochen, Germany \\ ** Carl Zeiss NTS GmbH, 73446 Oberkochen, Germany \\ *** Aalen University, Materials Research, 73430 Aalen, Germany
}

For a deeper understanding of material characterization and failure analysis information from different microscopy imaging techniques is commonly required. Especially light microscopy (LM) and scanning electron microscopy (SEM) with different analytical methods are well established and complement each other in many aspects so that they are often used in combination [1]. LM can be used for a fast sample overview followed by high resolution imaging in SEM. Furthermore the different contrast mechanisms of color, polarization, and dark field imaging in LM combined with backscattered electron imaging, energy dispersive x-ray spectroscopy or electron backscattered diffraction mapping in SEM offer versatile beneficial possibilities for material analysis which cannot be utilized by one technique only.

However, due to the different contrasts in both techniques it is very difficult and time-consuming to find one and the same sample area in the SEM as observed in the LM and vice versa, which is often required by the workflow. To overcome these challenges, Carl Zeiss has introduced a novel and comprehensive solution featuring a new range of sample holders and a powerful software. The holders, which enable a correlative workflow, can be easily adapted to LM as well as SEM and contain a set of special marks which allow to reference to a microscope independent sample based coordinate system. Additionally the microscope software supports fast and automated definition and retrieval of sample regions among the different microscopy tools. The obtained images can easily be registered and visualized correlatively.

In the following the correlative characterization of Li-Ion battery materials is demonstrated. These batteries are attractive candidates for mobile electronic devices and automotive applications. The functionality of Li-Ion batteries is based on the change of active materials due to diffusion processes [2,3]. Parameters of cell performance and various aging mechanisms strongly depend on the appearance and changes in the microstructure. Therefore the investigation and quantification of microstructures in LM and EM is of high importance for the understanding of battery materials as well as for failure analysis. It is essential to characterize the identical region of interest with both microscopes to correlate information from the LM (geometry, optical appearance of phases, damaging effects) with the visualization and analysis techniques of the EM for more detailed results regarding the microstructure (particle size, shape and chemical composition). This technique allows detecting of artifacts, defects and material changes due to aging. In this contribution we will demonstrate that the efficient combination of these microscopic techniques and allocated analysis techniques form a viable basis for short time to result in failure analysis. 
References

[1] S. Amelinckx et al., Handbook of Microscopy, Viley-VCH, 1997

[2] D. Linden, Handbook of Batteries, McGraw-Hill Professional, 2001

[3] G.A. Nazri et al., Lithium Batteries : Science and Technology, Springer, Berlin, 2009

[4] We thank Carl Zeiss MicroImaging GmbH for their kind support.
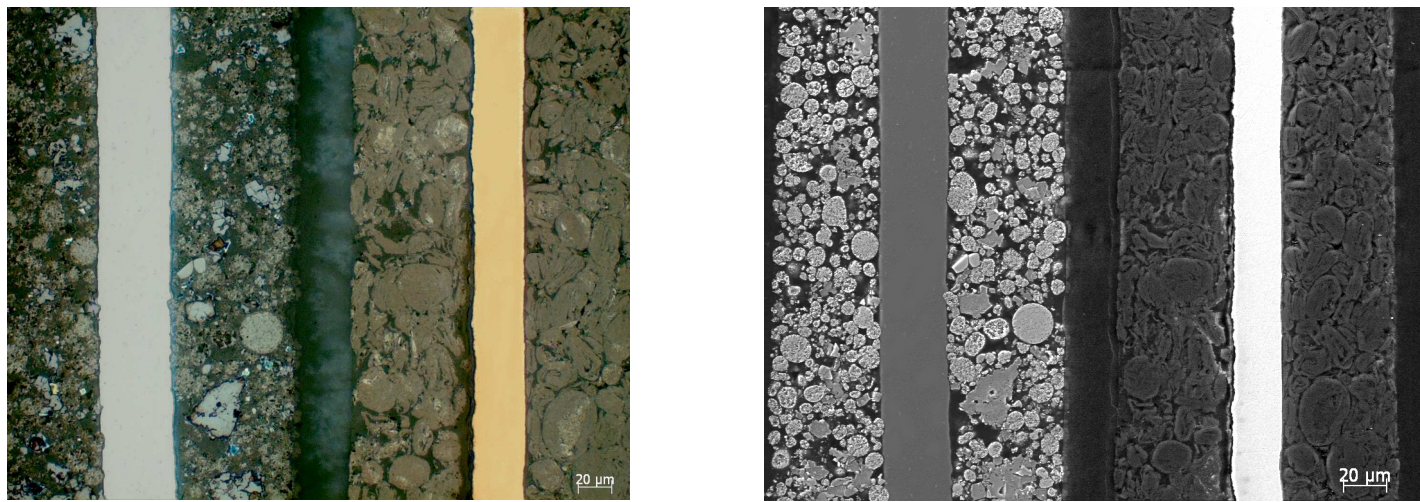

FIG. 1. CLEM of cathode, separator and anode of a Li-Ion battery type 18650 with brightfield LM (1) and backscattered electron contrast (r)
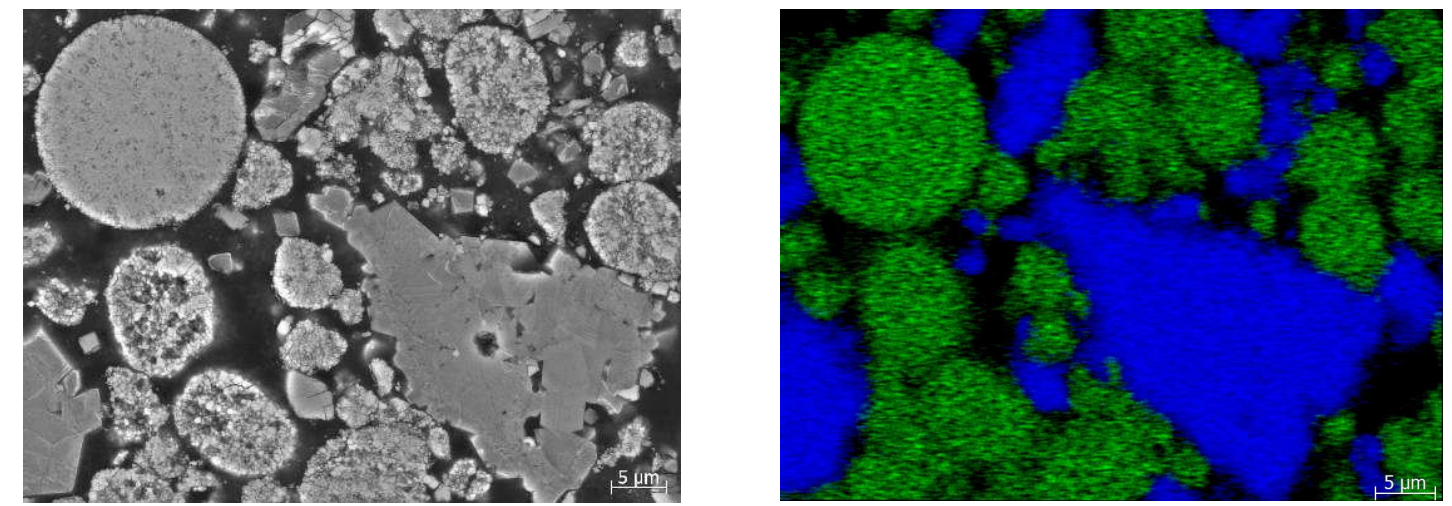

FIG. 2. SEM of cathode active material of a Li-Ion battery type 18650 with backscattered electron contrast (1) and EDX-Mapping on selected particles for identification of $\mathrm{LiMn}_{2} \mathrm{O}_{4}$ (blue) and $\mathrm{LiNi}_{\mathrm{x}} \mathrm{Co}_{\mathrm{y}} \mathrm{O}_{2}$ (green) 